\title{
Computer-aided design and Computer-aided engineering
}

\author{
Alexander Kolbasin ${ }^{1, *}$, Oksana Husu ${ }^{1}$ \\ ${ }^{1}$ Moscow State University of Civil Engineering, 129337, Yaroslavskoye shosse, 26, Moscow, Russia
}

\begin{abstract}
In modern industrial production some of the major factors of successful development include: cost reduction of the production, improvement of its quality, as well as help to minimise the time in market entry. Computer-aided design and Computer-aided engineering (CAD / CAE - systems) are the most effective for implementation of these requirements. Possible use of this engineering modeling simulation in conjunction with the power and speed of high performance computing could reduce costs and time of each cycle of designing, and also significantly reduce development time. The introduction of new technologies, the use of high quality products and engagement of qualified personnel would allow businesses and organizations to get on a path of innovative development of design and production systems.
\end{abstract}

\section{Introduction}

Computer-aided design (CAD) is a technology, the task of which is to facilitate creation, change, analysis and optimization of projects at the expense of automation of works on design stages and preparations of production. Any application working with computer graphics or using in calculations belongs to systems of Computer-aided design. Leading three-dimensional CAD-systems can enable realization of ideas of «door-to-door» preparation cycle, as well as the production of complex industrial products [1, 2, 3].

Civil engineers have been using computers for their calculations for a very long time. Digital computers have already been used for analyzing the electrical system or optimizations in prototype «Vortex» in 1949 year. Designers of early computers created utility programs, so that programmers can monitor applications by using block-schemes on screen with logical switches that can be open and close during a session in debug mode. They have been able to create electronic symbols and geometric diagrams that can be used for creation of simple overarching framework and flow charts. It was followed by pleasant discoveries that once painting object can optionally be replicated or amended. Other elaborations were held in 1960s in aviation, automotive and electronics applications in the field of 3D surface construction, programming of Numerical control and analysis of design $[4,5]$.

\section{Materials and methods}

${ }^{*}$ Corresponding author: alex123456789.a@yandex.ru 
Invention of 3D CAD / CAM was made by French Engineer - Pierre Bézier. After his mathematics work about surfaces, he developed «UNISURF» in the period from 1966 to 1968 to facilitate designing components and tools for the automotive industry. Then «UNISURF» became a working base for future generations of CAD.

The turning point was development of «SKETCHPAD» system in MIT by Ivan Sutherland. Distinguishing feature of «SKETCHPAD» was that it allowed designer to graphically interface with computers: design could be integrated with computer, painting on CRT monitors with pen-based technology. Basically, it was a prototype of graphical user interface, which cannot be substituted by modern CAD.

In the 1970s CAD-systems were limited to the production of sketches looked like handmade ones. The achievements in the field of programming and computer equipment, in particular developments in solid modelling in the 1980s, allowed more versatile use of computers in the design.

In 1981 John Walker started the company «Autodesk», which resulted in the creation of a 2D system of AutoCAD. The next step was production of «Pro/ENGINEER» in 1987, which marked wider using of modeling techniques based on the target attribute and parameter coordination. Also, the development of a solid modeling kernel (engines for manipulating 3D-objects), files Parasolid (ShapeData) and ACIS (spatial technologies) were of great importance for the development of CAD systems at the end of $1980 \mathrm{~s}$ and the beginning of 1990s. This resulted in output of packages of the average range, such as «SolidWorks» and «TriSpective» (later known as «IRONCAD») in 1995, «Solid Edge» (than «Intergraph») in 1996 and «Autodesk Inventor» in 1999 [6].

The main function of Computer-aided design (CAD) is to define the shape of structures, since further stages in development of the product's life cycle are defined exactly by geometry. Usually, systems of the development of working sketches and systems of geometrical simulation, which relate to the systems of automated designing, are applied.

CAD is widely used in many branches of industry, including automobile, shipbuilding and aerospace industry, architectural and industrial design, prosthetics, etc. Because of its huge economic importance, CAD was the main driving force for research in the area of computational geometry, computer graphics (both hardware and software) and discrete differential geometry [7].

Current software packages for Computer-aided design vary from 2D vector editing systems to 3D solid-state and surface modelers. Modern packages of CAD may also often allow rotations of the object in three dimensions, allowing viewing the designing object at any angle. Some CAD software is capable of dynamic mathematical simulation.

Computer-aided design is one of the main tools used by the engineers and designers. Currently, to develop various products, industrial enterprises widely use the following computer technologies - automation software:

- CAD/CAM-systems (Computer-Aided Design / Computer-Aided Manufacturing) which provide an integrated solution to the tasks of design and technological designing. Among the whole variety of CAD / CAM-systems, the most widely represented on the market, the following can be allocated:

- "heavy systems" ("3-D high-end") which appeared in 1980s and has wide range of functionalities and high performance: «CATIA» from «Dassault Systèmes», «NX» and «Creo Elements».

-“medium systems" (“3-D middle range") - primarily it is «SolidWorks», «Solid Edge», "Autodesk Inventor», in which, when they appeared in the mid-1990s, the possibilities of 3-D solid modeling were combined with the low price (in comparison with "Heavy" sys- 
tems) and orientation to the Windows platform. Among domestic CAD / CAM systems of this level - KOMPAS and T-Flex;

-"light systems" (2-D systems) which are the most common products of automation for designing, among the many of which, first of all, should be named AutoCAD.

- CAE-systems (Computer-Aided Engineering) are the systems of automation of engineering calculations, the most advanced of which represent multi-disciplinary suprasectoral CAE-systems.

$\mathrm{CAD}$ is also used to accurately create photo-modeling, which is often required in the preparation of environmental reports in which the projected building structures are superimposed on photographs of existing conditions where the intended objects can be built.

$\mathrm{CAD}$ is used for designing of various details and machines, as well as at the development and designing of all types of buildings, from small dwellings to the largest commercial and industrial structures (hospitals and factories).

CAD-systems have become particularly important within automated technologies because these systems have lower costs of product development and considerably reduced design cycle. Originally, software for the Computer-Aided Design was developed in such languages as Fortran, but with the development of methods of object-oriented programming, everything changed. Typical modern parametric functions are built around a number of key modules with their own APIs.

Currently, CAD-systems exist on all major platforms, including Windows, OC Linux, Unix and macOS. ArchiCAD and Vectorworks work on Windows and macOS, but not on Linux. For example, AutoCAD works only on Windows.

Computer-aided engineering is the complex of programs which include technologies serving in computer systems for working on analysis of CAD geometry, for creation of the product model and studying its behavior in reality, and also for improving and streamlining its structure. In CAE-systems, three-dimensional model of the object is used and created in the CAD-system. Most popular Computer-aided engineering systems - T-FLEX Analysis, APM WinMachine 2010, APM Civil Engineering 2010, ANSYS, etc.

CAE software is used on different types of the computer, such as mainframes, technical stations, and even personal computers. The choice of a computer system is often dictated by the computational power that is required to apply the CAE or the desired level (and speed) of the graphics interaction.

Design-Engineers use a great number of CAE tools, including large-scale commercial programs of general-purpose and many specialized programs written «in-house» or in other branches. Solution of engineering task frequently requires a few CAE tools. Data communication between different software is problematic for the most applications. Data are usually transferred through their own neutral formats of files, data exchange standards or system database.

A typical program of CAE consists of a number of mathematical models coded by algorithms written on the programming language. Analyzed natural phenomena are presented by engineering model. Physical configuration is described by geometrical model. The results, together with geometry, are visible through the user interface on display and the model of transition (displays of graphs).

\section{Results}

Computer-aided engineering make it possible to evaluate how the object is operable, using small material and time costs.

Using CAE-systems, the following is possible to be carried out:

- Analysis of components and groups using the Finite element method; 
- Thermal and hydrodynamic analysis;

- Kinematic researches;

- Modeling the processes, for example, die casting;

- Streamline processes or products;

- In general, there are three stages to deal with any automated tasks:

- Preliminary processing - definition of model and environmental factors which will be applied to it;

- Analysis (usually performed on powerful computers)

- Post processing of results (using visualisation tools)

This cycle is repeated (usually many times) either manually, or using commercial optimization software.

One of the most common methods of computer analysis is the Finite Element Method. This method simplifies the tasks on calculation of deformations, stresses, heat transfer, magnetic field distribution and other tasks with continuous media. Finite Element Method allows the connection of elements to be represented as an analytical model which can be segregated into separate parts. The computer can read these simplified parts and process them.

In the Finite Element Method, the reference design is replaced by an abstract model. Difference between an abstract model and structure is that an abstract model is formed by eliminating non-existent parts and reducing dimensions. A model can be created both automatically and interactively. Then, the finished analytical model is divided into finite elements which subsequently form an analytical model. The designing of an abstract model and the division of it on separate parts occur using software called preprocessors. After analyzing each element, computer connects all results together and outputs them in a visual format. Visualization software tools - postprocessors [8].

Computer-aided Engineering (CAE) belongs to industrial technology, which are specifically designed to the most important fields for equipment production that, undoubtedly, specifies them among other applications. Today, without the use of CAE-systems it is impossible to produce complex science-intensive products (ships, planes, tanks, various industrial equipment, etc.). CAE-systems provide unified support for the entire development cycle: from conceptual design to technological preparation of production, its testing and maintenance.

New technologies, high-quality equipment and qualified personnel are necessary for modern industry. It is the combination of these components that will allow enterprises and organizations to enter the innovative path of development of design and production [9].

Primarily, the systems of Computer-aided engineering help to reduce the costs for fullscale experiment and prototyping. Also, these systems make it possible to shorten the term for the output of finished products to the market. This acceleration occurs due to the absence of the need for a multiple iterative process of full-scale testing and modification of the design according to the results of their tests. The engineer has the opportunity to "play" the parameters and properties of the structure, to achieve optimal parameters and properties of the designed product $[10,11]$.

With the help of CAE-technologies it becomes possible to use inter-industry and interdisciplinary transfer of methodologies and technologies by storing, accumulating and sharing the experience of engineers and designers. Therefore, it is advisable to carry out engineering computational analysis at the earliest stages of designing and constructing products [12].

\section{Discussions}


The main areas in the development of CAE-systems:

- Improvement of methods for solving the interdisciplinary tasks of simulation;

- Development of platforms for integration of various systems of CAE;

- Increase of interoperability CAE and CAD systems;

- Improvement of the model's characteristics that are used to describe properties of materials;

- Improvement of the conditions for simulation of complex structures with a large number of degrees of freedom.

CAE-systems were proliferated in automobile and aircraft construction, electricity and electronics, heavy engineering and military industries. In 2012 North America became the biggest geographical region because CAE became popular, and the leading position on the growth rate was taken by the Asia-Pacific region, which is actively developing the industry $[13,14]$.

In order to complement the multidisciplinary industry-specific CAE-systems from the point of view of knowledge management, specialized PDM-systems were developed: Engineering Knowledge Management (EKM), Simulation Lifecycle Management (SLM); Simulation Process Management (SPM); Product Simulation Management (PSM).

In the CAE world market, leading positions are held by such large companies as «Ansys», «MSC Software», «Dassault Systemes», «CD-adapco Group» and «LMS International». In addition to them, there are many smaller companies working in the development of CAE-systems, but the number of such companies is declining as they are bought by larger players for the sake of their developed technologies.

Currently, some CAE and PLM suppliers have started to promote "globalized" licenses. By purchasing such licenses, customers can use CAE systems anywhere in the world and apply for support services to the supplier's office in any country. Such innovation will allow vendors to reduce the difference in costs of products in different countries, i.e. it will be possible to set the unified price to products worldwide. It is expected that more and more suppliers of CAE and PLM will apply this approach. Then there will be significant changes in the world market in terms of pricing policy of vendors [15].

Even though CAE-systems have a good reputation as the tool for testing, debugging and analysis, it is still believed that in the cycle of designing sufficiently accurate results come pretty late. It could be a problem because modern products become more and more complex. They include smart systems, which leads to an increase in the need for multi-physics analysis, including control elements, and new light materials, with which engineers are not familiar. Companies and producers of CAE software are always looking for the ways to improve the process to change that situation. It is necessary to develop more powerful solvers, better use the computer resources and include technical knowledges in pre - and postprocessing $[16,17]$. Also, one needs to achieve a better alignment between the simulation of «3D CAE» system, «1D» system and physical tests. This should ensure an increase in the realism of simulation and speed of calculation. In addition, the integration of CAE into overall product life cycle management should be improved. Thus, companies and producers of CAE software can combine design of the product with benefit of the project.

\section{Conclusions}

CAE-systems are powerful tools for engineering analysis, which are successfully applied to solve a variety of practical tasks. Computer-aided design is the standard in almost any industry that uses some software to develop products. CAE is the next step not only in product development, but also in supporting of the engineering process, because it allows performing tests and simulation physical properties of the product without a physical proto- 
type. By using these advantages of Computer-aided design, especially in combination with power and speed of high-performance computing, the cost and time of each design iteration cycle, and also the overall development process can be significantly reduced.

\section{References}

1. A.I. Borovkov, Computer-Aided Engineering (Publisher SPbSTU, St. Petersburg, 2012)

2. M.V. Golovitsyna, Intelligent computer-aided design systems (National Open Institute, 2016)

3. A.I. Kondakov, CAD of technological processes and production (ACADEMA, 2007)

4. A.O. Vylegzhanina, Information-technological and software of control of project: educational supplies (Direct-Media, Berlin, 2015)

5. Y.Z. Pastukhova, Computer graphics in construction (NRU MGSU, Moscow, 2016)

6. A.M. Kolbasin, A.V. Ilyukhin, V.I. Marsov, The ways of creation of effective automated control system of production of building materials (MADI, Moscow, 2016)

7. A.V. Ginzburg, Computer-aided design in construction: educational supplies for university students studying on 27.08.00. "Construction» (MGSU, Moscow, 2014)

8. V.H. Malukh, Introduction to modern CAD: course of lectures (DMK press, Moscow, 2010)

9. I.P. Norenkov, Basis of Computer-aided design: educational supplies for universities. 4-th edition, republishing the advanced and added volume (Publisher Bauman MSTU, Moscow, 2009)

10. D.M. Ushakov, Introduction to mathematical foundations of CAD (DMK Press, Moscow, 2012)

11. V.J. Sudzilovsky, Modelling and algorithmization in CAD (Book club 36.6, Moscow, 2009)

12. Z.K. Sabonnadiere, The Finite Element Method and CAD (Book on demand, Moscow, 2012)

13. P.N. Latvians, CAD catalog. Programs and manufacturers (Solon-Press, Moscow, 2010)

14. O.D. Goldberg, I.S. Sviridenko, Engineering design and CAD of electric machines (Academia, Moscow, 2008)

15. M.V. Bakanov, V.A. Liska, V.V. Alekseev, Data systems (UNITY, Moscow, 2010)

16. M. Abdulkhanov, V.A. Vorobyov, The technology of production of materials and articles and automation of process routes on the enterprises of highway engineering: educational supply (Solon-press, Moscow, 2014)

17. S.I. Yevtushenko, V.A. Vorobyov, D.Y. Parshin, Automation and robotic of construction: educational supply (IC RIOR, NIC INFRA-M, Moscow, 2014) 